\title{
Transcriptome analyses identify key genes and potential mechanisms in a rat model of osteoarthritis
}

\author{
Hui-Zi Li $i^{1,2}$ and Hua-Ding Lu, ${ }^{1,2^{*}}$
}

\begin{abstract}
Background: Osteoarthritis $(\mathrm{OA})$ is one of the most common degenerative diseases of the joints worldwide, but still the pathogenesis of OA is largely unknown. The purpose of our study is to clarify key candidate genes and relevant signaling pathways in a surgical-induced OA rat model.

Methods: The microarray raw data of GSE8077 was downloaded from GEO datasets. GeoDiver were employed to screen differentially-expressed genes (DEGs). Enrichment analyses of DEGs were performed using Metascape. Construction of protein-protein interaction (PPI) network and identification of key genes were conducted using STRING, Cytoscape v3.6.0, and Centiscape2.2. Furthermore, miRDB and Cytoscape v3.6.0 were used for visualization of miRNA-mRNA regulatory network. Kyoto Encyclopedia of Genes and Genomes (KEGG) pathway analysis for predicted miRNAs was undertaken using DIANA-miRPath v3.0.

Results: Several DEGs (188 in comparison between OA and sham-operated group and 160 in comparison between OA and contralateral group) were identified. DEGs mainly enriched in vasculature development, regulation of cell migration, response to growth factor (Gene ontology), and ECM-receptor interaction (KEGG). Two comparison cohorts shared 79 intersection genes, and of these, Ccl2, Col4a1, Col1a1, Aldh1a3, and Itga8 were defined as the hub genes. Predicted miRNAs of seven DEGs from sub-networks mainly enriched in MAPK signaling pathway.

Conclusion: The current study shows that some key genes and pathways, such as Ccl2, Col4a1, Col1a1, Aldh1a3, Itga8, ECM-receptor interaction, and MAPK signaling pathway may be associated with OA progression and act as potential biomarkers and therapeutic targets for OA.
\end{abstract}

Keywords: Osteoarthritis, Differentially expressed genes, Animal model, Bioinformatics analysis

\section{Introduction}

Osteoarthritis (OA), one of the most important causes leading to joint disability, is associated with increased social and medical burden [1,2]. Regardless of wonderful advancements in diagnosis and treatment of $\mathrm{OA}$ these years, the prevalence of OA still increases from $6.6 \%$ to 14.3\% between 1999 and 2014 in the USA [3, 4]. Currently, the main management for early-stage OA includes lifestyle modification and pharmaceutical drugs,

\footnotetext{
* Correspondence: johnniehuading@163.com

'Department of Orthopaedics, The Fifth Affiliated Hospital of Sun Yat-Sen University, Zhuhai 519000, Guangdong, China

${ }^{2}$ Guangdong Provincial Engineering Research Center of Molecular Imaging,

The Fifth Affiliated Hospital of Sun Yat-Sen University, Zhuhai 519000,

Guangdong Province, China
}

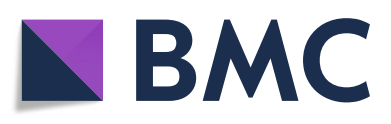

(c) The Author(s). 2018 Open Access This article is distributed under the terms of the Creative Commons Attribution 4.0 International License (http://creativecommons.org/licenses/by/4.0/), which permits unrestricted use, distribution, and

reproduction in any medium, provided you give appropriate credit to the original author(s) and the source, provide a link to the Creative Commons license, and indicate if changes were made. The Creative Commons Public Domain Dedication waiver (http://creativecommons.org/publicdomain/zero/1.0/) applies to the data made available in this article, unless otherwise stated. anti-inflam and coricoseroid injections [5-9]. and corticosteroid injections [5-9]. Irrespective of their potential effectiveness in increasing the time from diagnosis of $\mathrm{OA}$ to joint arthroplasty, these non-operative treatments can hardly block or reverse OA progression [10]. Eventually, total joint replacement was recommended by orthopedic surgeons for patients with advanced OA owing to serious radiographic grade, pain, and functional impairment of involved joints [11]. A crucial reason for these lies in that the key candidate genes and relevant signaling pathways associated with OA remains largely unknown. As a result, it is critical to further elucidate the pathogenesis of $\mathrm{OA}$ onset and progression.

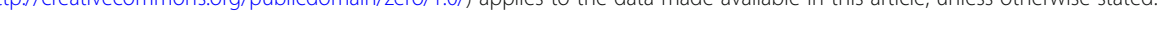


Accumulative evidence suggested that many differentially expressed genes (DEGs) may participate in OA development. Kuttapitiya and colleagues demonstrated that 218 DEGs in bone marrow lesions of OA patients were related to OA-induced pain [12]. Ramos et al. suggested that 694 DEGs were identified in blood of OA patients and these DEGs mainly enriched in the apoptosis pathways, which may be associated with the onset of OA [13]. Recently, bioinformatics analysis were widely used to identify DEGs and perform subsequent enrichment analyses, such as Gene ontology (GO) and Kyoto Encyclopedia of Genes and Genomes (KEGG) pathway analysis, which may largely promote the understanding of $\mathrm{OA}$ pathogenesis $[14,15]$. Surgical-induced OA rat model which mainly involves anterior cruciate ligament transaction and destabilizing medial meniscus, is frequently used to explore the pathogenesis of OA in vivo. Previous studies also indicated that several ectopically expressed genes, such as AQP-1, GDF5, and TAK1, participated in the development of surgically induced OA in rat models [16-18]. However, most of researchers merely attached importance to individual OA-related gene, which can hardly have a comprehensive understanding of corresponding molecule mechanisms, which were usually complicated and networked. Understandably, bioinformatics analysis may be a powerful way to explore these complicated regulatory networks and molecule mechanisms in a surgical-induced OA rat model.

In the current study, we identified several DEGs in a surgical-induced $\mathrm{OA}$ rat model after re-analyzing the raw microarray data (GEO Series: GSE8077). Enrichment analyses of DEGs were performed using Metascape. Construction of protein-protein interaction (PPI) network and identification of key genes were conducted using STRING, Cytoscape, and Centiscape2.2. Furthermore, miRDB and Cytoscape v3.6.0 was used for visualization of miRNA-mRNA regulatory network. We also performed KEGG pathway analysis for predicted miRNAs based on DIANA-miRPath v3.0.

\section{Materials and methods Microarray data}

The microarray data (GSE 8077 or GDS2809) deposited by Appleton et al. was downloaded from GEO database (https://www.ncbi.nlm.nih.gov/geo/), which included three groups (surgical-induced OA group, contralateral group, and sham group) [19]. The expression data was generated on Affymetrix Rat Genome 2302.0 Array platform [Rat230_2]. In the dataset, five rat models of OA were established through anterior cruciate ligament transection and partial medial meniscectomy. Another five rats were performed with sham surgery to act as control groups. After 4 weeks of forced mobilization, three times per week, cartilage of three groups was harvested for further experiments. Surgical-induced OA rat models were successfully induced at 4 weeks after operation, which were verified by Safranin O staining [19].

\section{Identification of differentially expressed genes}

DEGs between OA and control group (Contralateral group or Sham group) were identified using GeoDiver (https://www.geodiver.co.uk/). Briefly, two comparison groups(OA vs. Contralateral group or Sham group) were assigned to analyze after loading the data of GDS2809 in GeoDiver. And then, DEGs were identified after clicking "ANALYSE GEO DATASET" with default parameters. DEGs with the cut-off criterion ( $\mathrm{P}$ value $<0.05$ and $\log |\mathrm{FC}|>1$ ) were considered for further analyses.

\section{Enrichment analysis for DEGs}

Enrichment analyses for DEGs were performed using Metascape (http://metascape.org/gp/index.html\#/main/ step1), a powerful web-based tool, which involved in four processes: ID Conversion, Gene Annotation, Membership Analysis, and Enrichment Analysis [20]. The available terms for enrichment analysis includes pathway (Reactome Gene Sets, Canonical Pathways, BioCarta Gene Sets, GO Biological Processes, Hallmark Gene Sets, and KEGG Pathway), functional set (Go Molecular Functions), structural complex (Go Cellular Components, KEGG Structural Complex, and CORUM Protein Complex), and signature module (immunologic signatures, oncogenic signatures, and chemical and genetic perturbations). More interestingly, Metascape provides more frequently updated bioinformatics analyses than DAVID [21]. Three steps were followed to perform enrichment analyses for DEGs in the current study. Firstly, we undertook enrichment analysis for DEGs in two comparison cohorts independently. And then, enrichment analysis for the intersection genes across two comparison cohorts was performed. Finally, we conducted meta-enrichment analysis for two DEGs lists.

\section{Integration of protein-protein interaction (PPI) network and module analysis}

PPI networks of intersection DEGs were analyzed with the threshold (combined_score $>0.4$ ) using the STRING tool (http://www.string-db.org), which can provide interactions across matched proteins [22]. And then, Cytoscape v3.6.0 was employed to construct PPI networks [23]. Cytoscape plug-in CentiScaPe was used to assess the centrality of DEGs in PPI networks with three algorithms: degree centrality, betweenness centrality, and closeness centrality [24]. DEGs with centrality degree $\geq 3$ were defined as key genes in the current study. Furthermore, the expression level of hub genes between sham group and OA group were compared with unpaired Student's $t$ test after extracting the original expression value in GEO Profiles (https://www.ncbi.nlm.nih.gov/geoprofiles/). $P<0.05$ was thought to be statistically significant. All the statistical 
analyses were conducted using GraphPad Prism 7. Subsequently, molecular complex detection (MCODE) was applied to extract sub-networks in PPI networks with default algorithms (degree cut-off of 2, node score cut-off of 0.2 , K-Core of 2, and max. depth of 100) [25].

\section{Construction of miRNA-mRNA regulatory network and identification of miRNA-associated pathways}

MiRNAs, a class of non-coding RNA with 20-22 nucleotides (nts), can bind to the 3'UTR regions of targeted mRNAs to induce translational repression or degradation of mRNAs [26]. In the current study, miRNAs interacting with seven mRNAs from sub-networks (Itga8, Col1a1, Col12a1, Col4a1, Ccl7, Mmp12, and Ccl2) were predicted using an online database (miRDB) [27]. Cytoscape was used for construction of miRNA-mRNA regulatory network. We also performed KEGG analysis for predicted miRNAs based on DIANA-miRPath v3.0, a useful web tool which can provide experimentally supported miRNAs-mRNA interaction [28]. The results of KEGG enrichment for predicted miRNAs were visualized using package ggplot2 in R (https://www.r-project.org/).

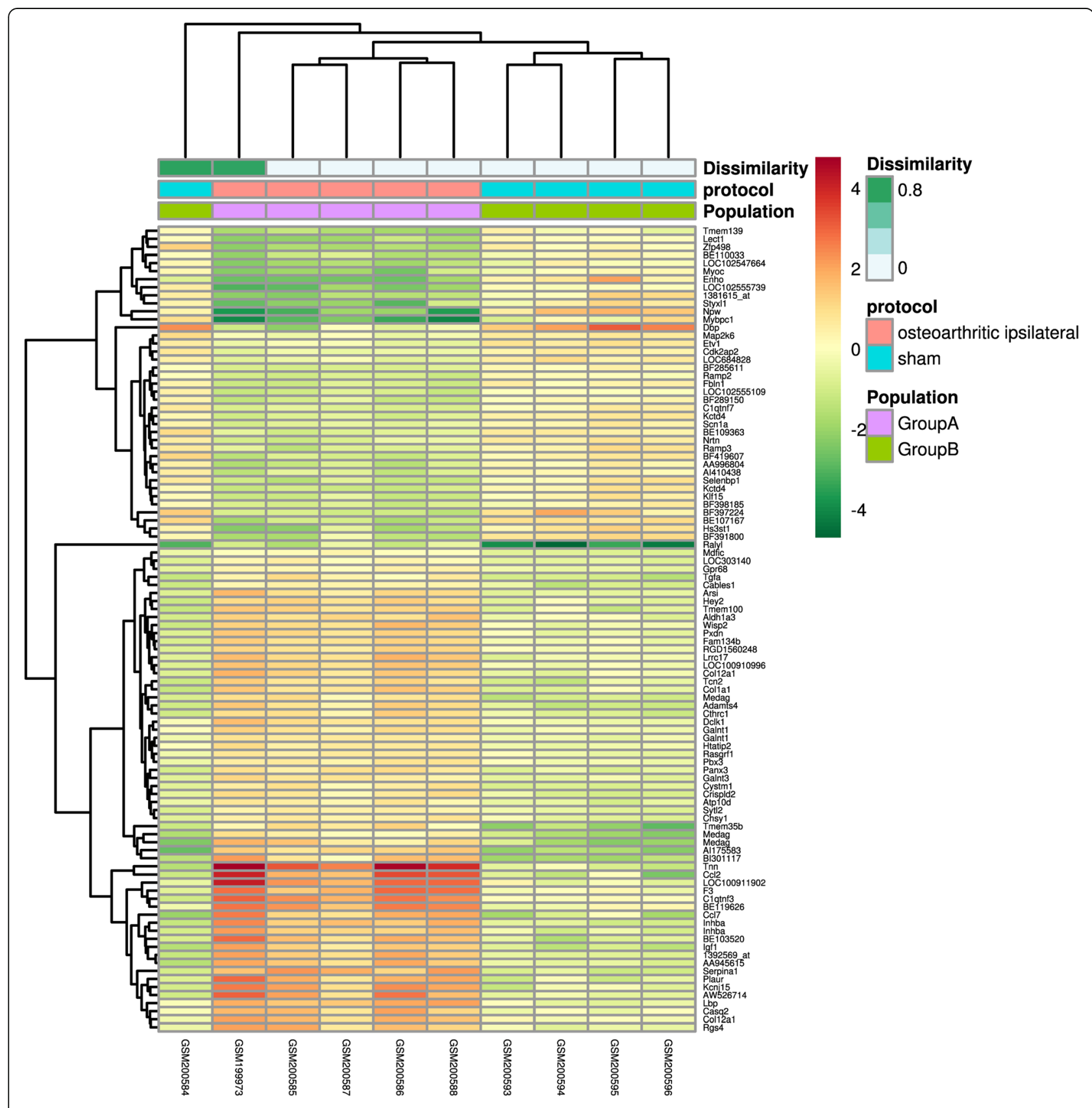

Fig. 1 Heatmap of DEGs in comparison between OA group and sham group. The heatmap was produced using GeoDiver(https://www.geodiver.co.uk/) 


\section{Results}

\section{Analysis of DEGs}

Several DEGs (188 in comparison between OA and shamoperated group, and 160 in comparison between OA and contralateral group) were identified using GeoDiver according to the pre-defined criterion. The results of heatmap were showed in Fig. 1 (OA vs. sham group) and Fig. 2 (OA vs. contralateral group). Detailed DEGs of both comparison cohorts were showed in Additional file 1: Table S1.

\section{Enrichment analysis of DEGs}

DEGs in comparison between OA and sham group mainly enriched in urogenital system development, regulation of vasculogenesis, vasculature development, response to growth factor, negative regulation of cell proliferation, skeletal system development (GO), and ECM-receptor interaction (KEGG) (Fig. 3a). Furthermore, DEGs in comparison between $\mathrm{OA}$ and contralateral group mainly enriched in muscle system process, positive regulation of cell migration, regulation of system process, response

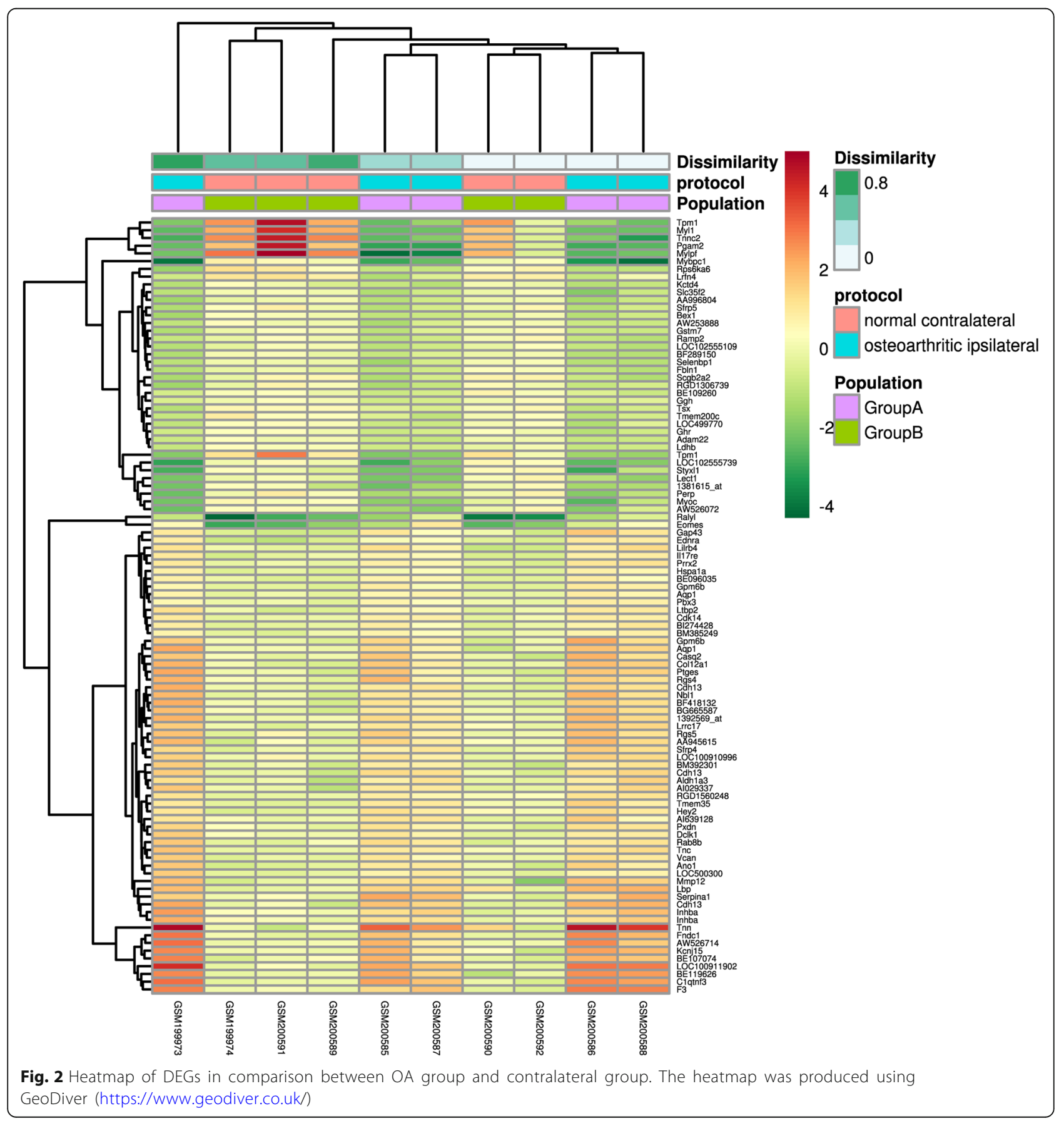




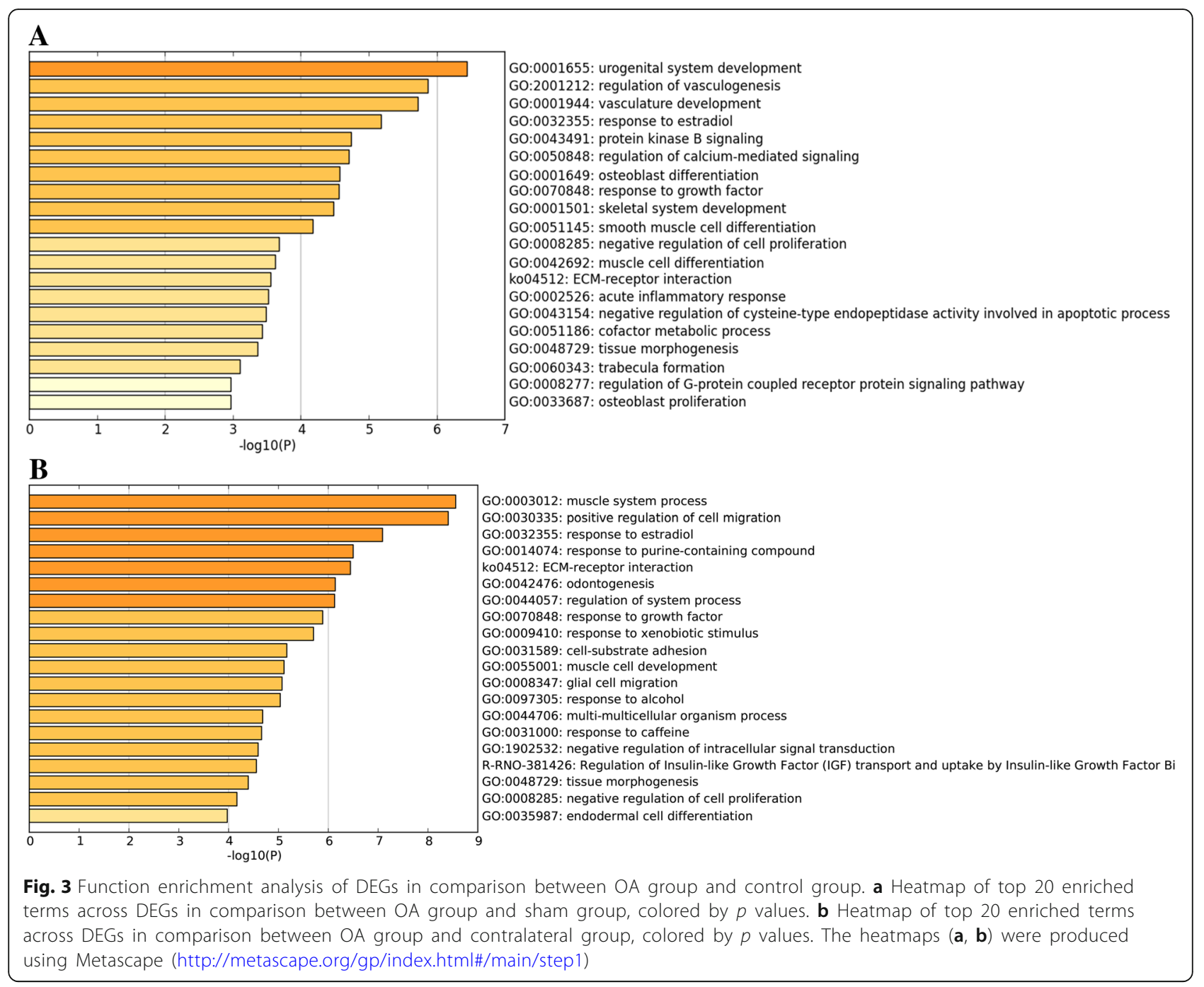

to growth factor (GO), and ECM-receptor interaction (KEGG) (Fig. 3b). Two comparison cohorts shared 79 intersection genes, which mainly enriched in positive regulation of cell migration, response to growth factor, negative regulation of cell proliferation, response to estradiol, cell-substrate adhesion (GO), and Extracellular matrix organization (Reactome Gene Sets) (Fig. 4a-c). Additionally, we also performed meta-enrichment analysis based on two DEGs lists, which suggested that DEGs from two comparison cohorts mainly enriched in vasculature development, positive regulation of cell migration, response to growth factor (GO), and ECM-receptor interaction (KEGG) (Fig. 5a-c).

\section{PPI network analysis of DEGs}

PPI network analysis of intersection genes contained 18 nodes and 19 edges (Fig. 6a). Centiscape 2.2 was employed to analyze the PPI network and five genes (Ccl2, Col4al, Col1a1, Aldh1a3, and Itga8) with centrality degree $\geq 3$ were defined as key genes (Table 1). Also, MCODE plugin was used to extract sub-network modules. Two subnetworks were screened out and contained 7 DEGs (Itga8, Col1a1, Col12a1, Col4a1, Ccl7, Mmp12, and Ccl2) (Fig. 6a). The expression levels of five hub genes in OA group were higher than those in sham group (Fig. 6b) (Additional file 2: Table S2).

\section{MiRNA-target regulatory network}

MiRNAs binding to DEGs in sub-networks were predicted using miRDB. The miRNA-mRNA regulatory network included 106 nodes and 96 edges. Of these, rno-miR-539-5p can antagonize both $\mathrm{Ccl} 2$ and Itga8 and rno-miR-199a-3p can antagonize both Ccl7 and Col12a1 (Fig. 7a). Also, we performed functional enrichment analysis of these predicted miRNAs, which mainly enriched in MAPK signaling pathway, pathways in cancer, proteoglycans in cancer, Hippo signaling pathway, and FoxO signaling pathway (KEGG) (Fig. 7b). 


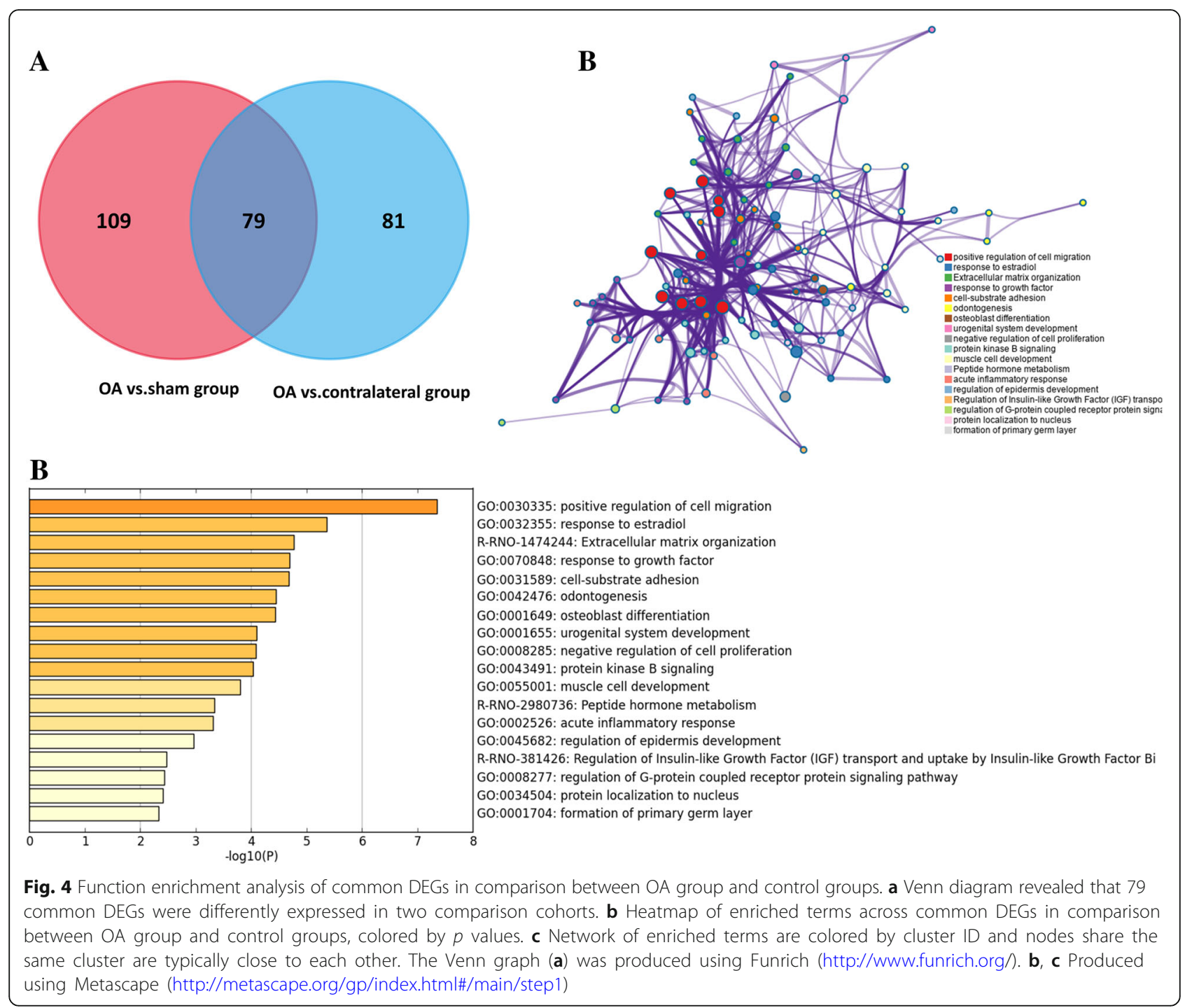

\section{Discussion}

The surgical-induced OA rat model is one of the most common animal models in vivo. Therefore, it is essential to unveil potential molecular mechanisms in a surgicalinduced OA rat model, which will contribute to clarifying the pathogenesis of OA. In the current study, several key genes and pathways were identified through reanalyzing GSE8077 dataset using integrated bioinformatics analysis.

Three steps were followed to perform enrichment analysis of DEGs, which revealed that DEGs mainly enriched in vasculature development, response to growth factor, positive regulation of cell migration, and ECM-receptor interaction. Many studies found that vasculature development at the osteochondral junction and synovium was associated with the onset and development of OA [29, 30]. Meanwhile, some growth factors, such as VEGF and NGF, were obviously upregulated in subchondral spaces, vascular channels and chondrocytes of OA patients [31]. Vasculature development was usually activated by some growth factors, such as VEGF [32]. Further studies also indicated that repression for angiogenesis in osteochondral junction and synovium may had a potential inhibitory influence on OA progression [33]. Accordingly, these growth factors may act as potential therapeutic targets for OA. Generally, loss of cartilage homeostasis and the dysfunction of chondrocytes phenotypes including cell apoptosis, cell migration, and cell proliferation are the critical pathological process of OA [34, 35]. The current study also revealed that DEGs enriched in positive regulation of cell migration and negative regulation of cell proliferation. Meanwhile, ECM-receptor interaction was found to be the most significantly enriched pathway for DEGs, which was further verified by many previous studies [36, 37]. Therefore, dysfunction of these cell phenotypes and molecules may play important roles in OA development and 


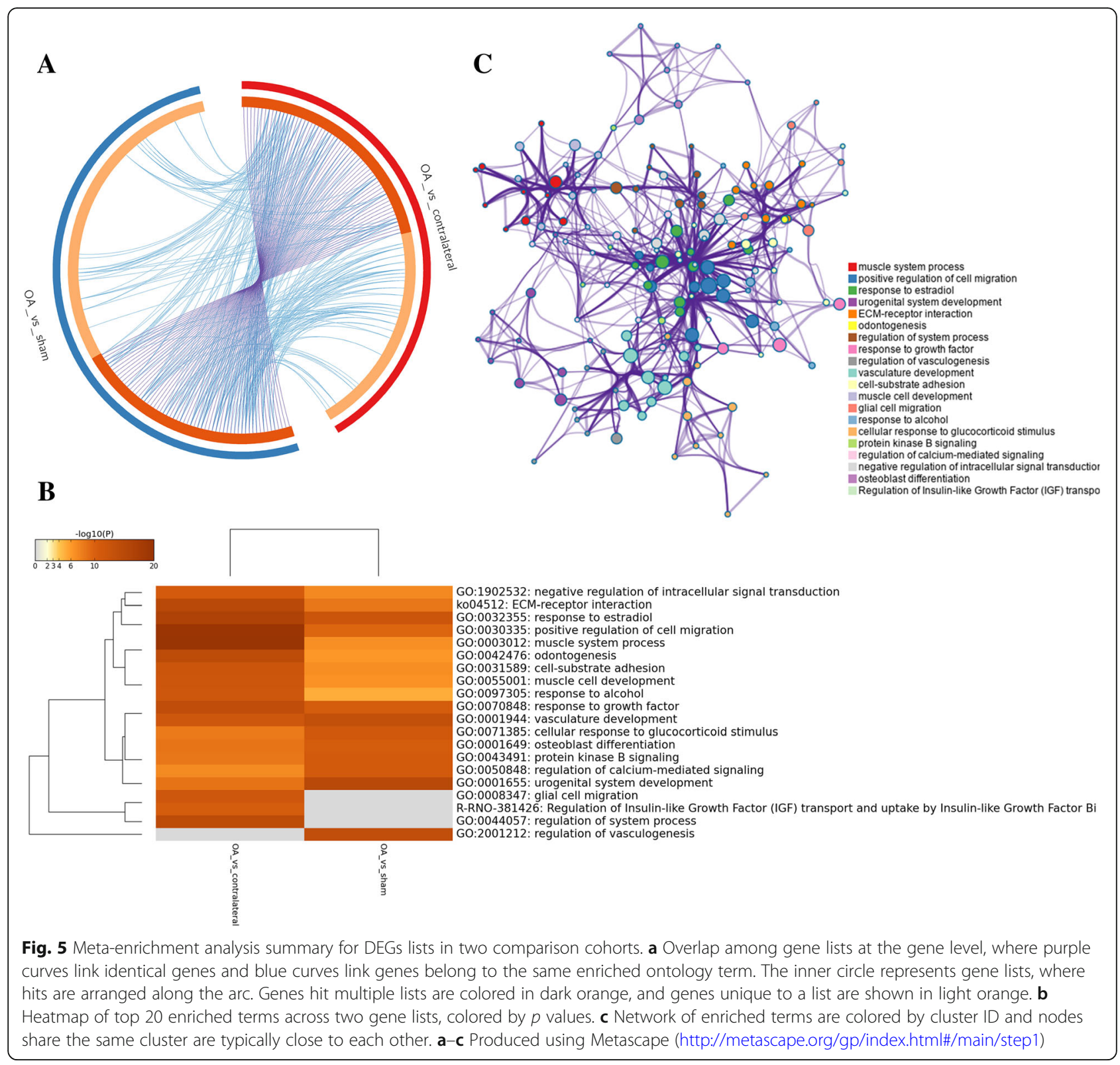

can act as promising pathological signatures for $\mathrm{OA}$ in vitro and in vivo.

Many OA-related key genes were also identified in the surgical-induced rat model, including Ccl2, Col4a1, Col1a1, Aldh1a3, and Itga8. Integrin $\alpha 8$ (Itga8) was an important component of ECM-receptor interaction pathway. It was significantly upregulated in mesenchymal cells and played important roles in the expression of extracellular matrix components [38]. Gong et al. revealed that Itga8 may participate in the degradation of extracellular matrix, including collagen type XI alpha 1, aggrecan, collagen type VI alpha 1 in periodontal ligament tissues [39]. Considering that imbalance of extracellular matrix anabolism and catabolism was the critical pathological process in OA, it was worthwhile to explore the potential roles of Itga8 in OA. Several studies revealed that the abnormal expression of collagen-related genes (Col1a1, Col4a1 and MMP12) participated in the pathogenesis of OA onset and progression, which were consistent with our study [40-43]. Our study also found that CCL2 was upregulated in surgical-induced rat OA model and may participate in OA pathogenesis, which was further supported by previous studies $[44,45]$. Recent studies revealed that CCL2 can be responsible for monocytes' migration and cartilage degeneration, and the CCL2/CCR2 axis may play a critical role in OA-related pain [46, 47]. As one of Aldehyde dehydrogenase isoforms, Aldh1a3 was obviously upregulated in human articular chondrocytes. 


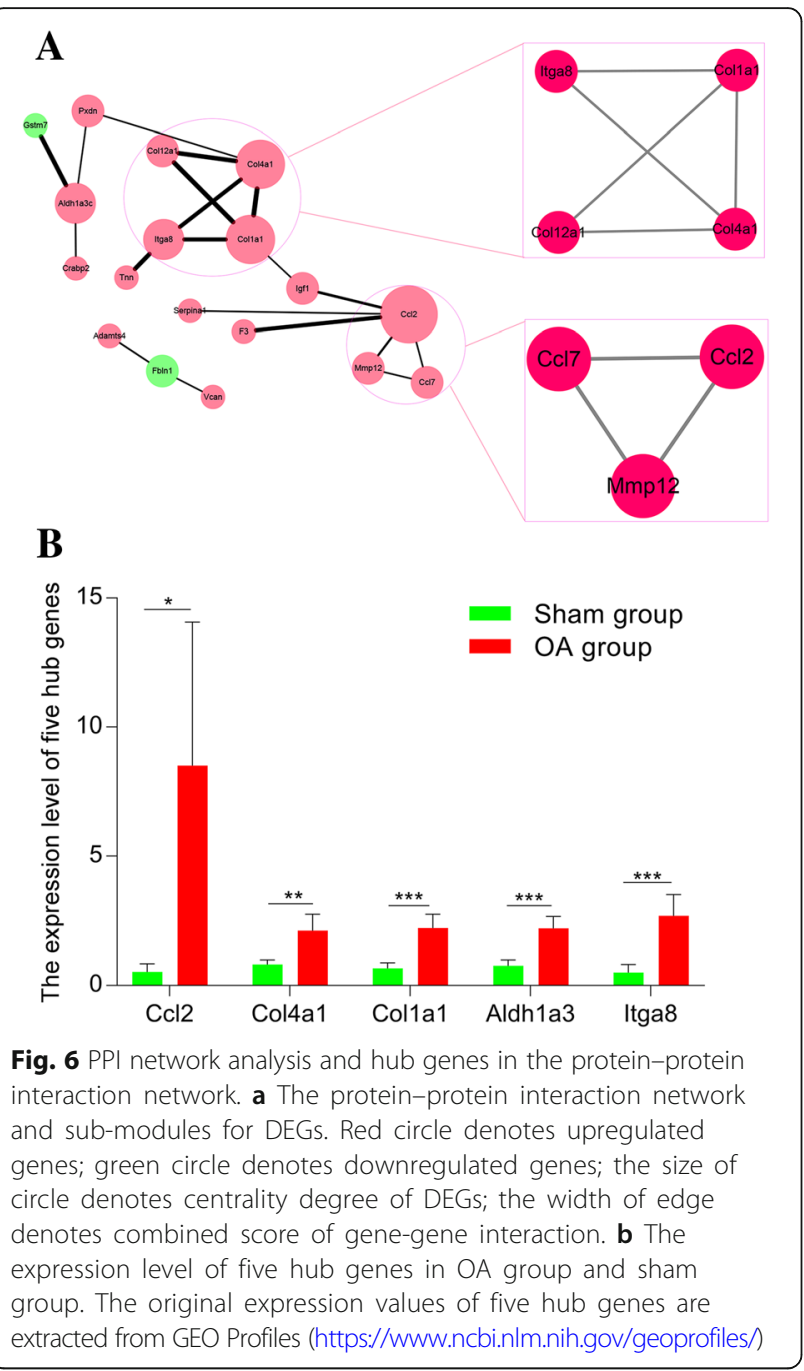

Furthermore, the activation of Aldh1a3 may be responsible for the producing activity of collagen II in chondrocytes [48]. Thus, whether the dysfunction of Aldh1a3 was associated with OA pathogenesis was worthwhile to be further explored. Collectively, considering that the important roles of these key genes in OA, they may be used as potential molecular biomarkers and therapeutic targets for OA.

Previous studies revealed that many differentially expressed miRNAs (for example, miR-145 and miR-140) were associated with $\mathrm{OA}$ development and progression $[49,50]$. Also, some researchers demonstrated that circulating miRNAs, such as miR-19b-3p, miR-122-5p, miR486-5p, hsa-miR-140-3p, hsa-miR-671-3p, and hsa-miR33b-3p, can be promising diagnostic biomarkers for knee OA $[36,51]$. In the current study, we identified that 99 predicted miRNAs were mainly enriched in MAPK signaling pathway and of these, miR-199a-3p and miR-539-59 may act as potential key miRNAs to regulate corresponding mRNAs. Actually, previous studies have verified that
Table 1 The statistical results of the connectivity degrees of the PPI network

\begin{tabular}{llll}
\hline Gene & Degree & $\begin{array}{l}\text { logFC (OA vs. } \\
\text { Sham group) }\end{array}$ & $\begin{array}{l}\text { adj.P.Val (OA vs. } \\
\text { Sham group) }\end{array}$ \\
\hline Ccl2 & 5 & 3.907 & 0.014 \\
Col4a1 & 4 & 1.331 & 0.026 \\
Col1a1 & 4 & 1.759 & 0.014 \\
Aldh1a3 & 3 & 1.53 & 0.015 \\
Itga8 & 3 & 2.598 & 0.019 \\
Col12a1 & 2 & 1.703 & 0.009 \\
Igf1 & 2 & 2.508 & 0.006 \\
Mmp12 & 2 & 1.879 & 0.026 \\
Fbln1 & 2 & -1.376 & 0.006 \\
Ccl7 & 2 & 3.02 & 0.015 \\
Pxdn & 2 & 1.266 & 0.026 \\
Gstm7 & 1 & -1.076 & 0.023 \\
Tnn & 1 & 4.65 & 0.005 \\
F3 & 1 & 2.545 & 0.009 \\
Adamts4 & 1 & 2.042 & 0.006 \\
Crabp2 & 1 & 3.089 & 0.011 \\
Serpina1 & 1 & 1.294 & 0.017 \\
Vcan & 1 & 1.13 & 0.023 \\
\hline
\end{tabular}

these predicted miRNAs and pathways played important roles in OA. For instance, Sun et.al. found that inhibition of P38-MAPK signaling pathway participated in repressing chondrocytes apoptosis and the release of proinflammatory cytokines in OA [52]. Furthermore, Akhtar et.al. suggested that overexpression of miR-199a can inhibit MAPK signaling pathway, thus attenuating OA progression [53]. Therefore, these important miRNAs and signaling pathways can be served as potential diagnostic biomarkers and therapeutic targets for OA, which may provide potential hallmarks for further experimental studies.

The strength of the current study was that we performed comprehensive enrichment analysis based on Metascape in a rat OA model. Apart from common DEGs between OA group and control groups, we also performed meta-enrichment analysis of all the DEGs in two comparison cohorts. Besides, pathway enrichment analyses were undertaken to explore the potential roles of predicted miRNAs. Regardless of aforementioned strengths, our studies also existed some limitations. Firstly, our findings were merely based on limited sample size (five in each group), so it was hard to exclude potential random error and false positive. Accordingly, further studies with large sample size should be warranted. Secondly, the results of the current study were totally based on bioinformatics prediction and lacked subsequent experimental verification, such as RT-qPCR, western blot, and immunohistochemistry. Actually, owing to limited available materials in 


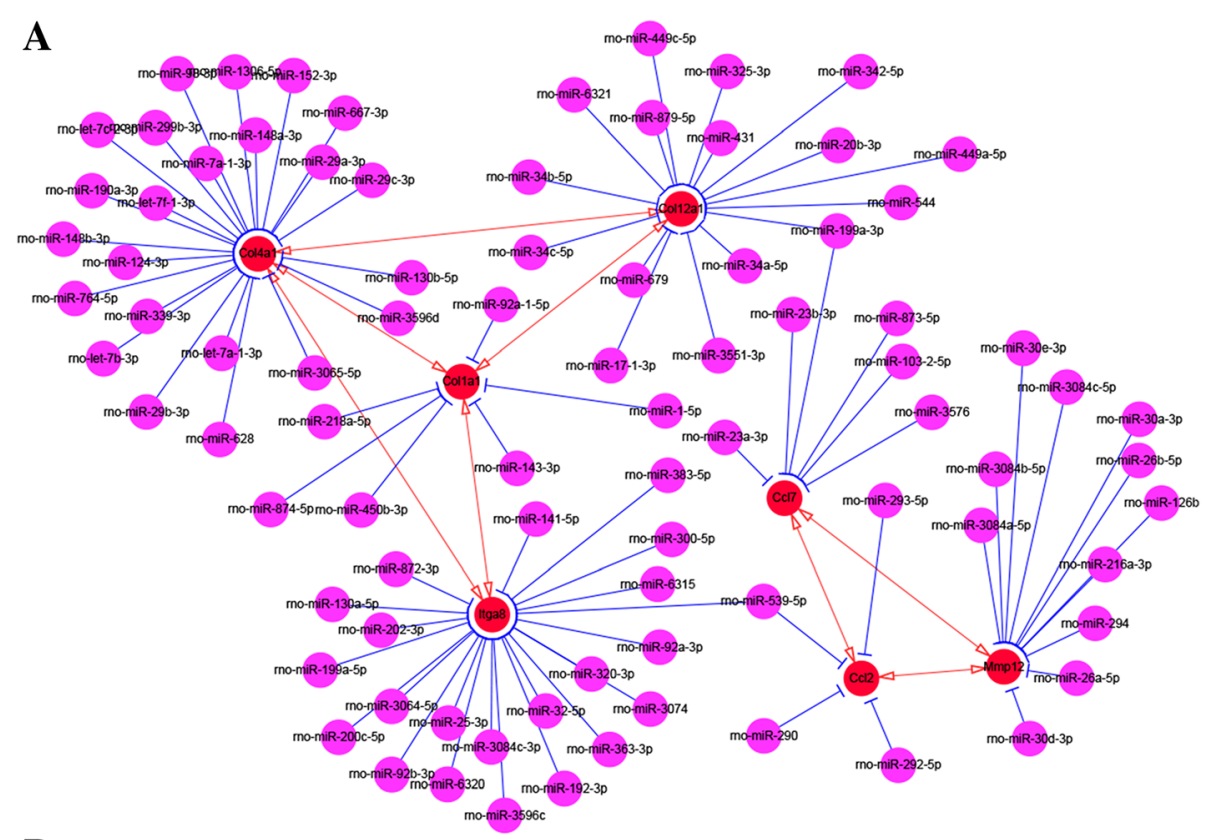

B

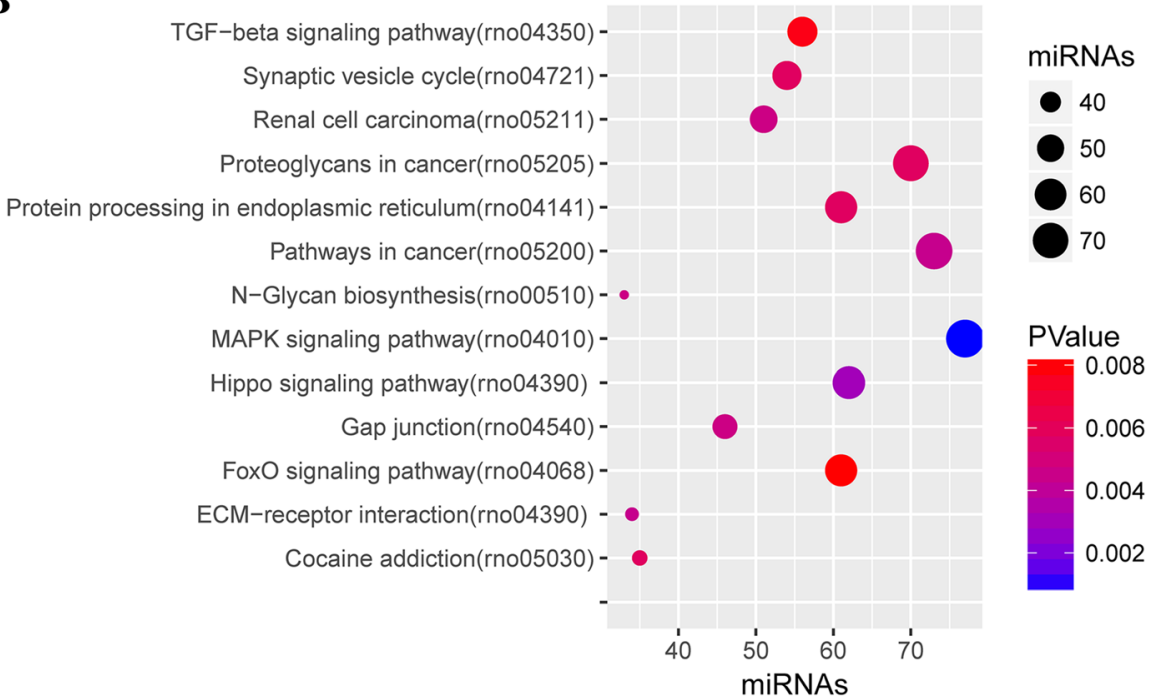

Fig. 7 MiRNA-mRNA regulatory networks in surgical-induced rat OA model. a MiRNA-mRNA (Itga8, Col1a1, Col12a1, Col4a1, Ccl7, Mmp12, and $\mathrm{Ccl} 2$ ) regulatory networks. Red nodes denote genes from sub-networks and purple nodes denote miRNAs; red edges denote mRNAs-mRNAs interaction relationship and blue edges denote miRNAs-mRNAs interaction relationship. b Bubble graph for KEGG pathway enrichment analysis of predicted miRNAs

the current study, it was hard for us to verify our findings with these experiments. Anyway, the current study may provide some potential useful orientation for future experimental studies.

\section{Conclusions}

The current study shows that some key genes and pathways, such as Ccl2, Col4a1, Colla1, Aldh1a3, Itga8, ECM-receptor interaction, and MAPK signaling pathway may be associated with OA progression and act as potential biomarkers and therapeutic targets for OA. These findings need further experimental verification, but may provide potential useful evidence for future researches in OA.

\section{Additional files}

Additional file 1: Table S1. Detailed DEGs in a surgical-induced rat OA model. (XLSX $12 \mathrm{~kb}$ )

Additional file 2: Table S2. The original expression value of five hub genes extracted from GEO Profiles. (DOCX $19 \mathrm{~kb}$ ) 


\section{Abbreviations}

DEGs: Differentially expressed genes; GO: Gene ontology; KEGG: Kyoto Encyclopedia of Genes and Genomes; MCODE: Molecular complex detection; PPI: Protein-protein interaction; RT-qPCR: Real-time quantitative PCR

\section{Acknowledgements}

Not applicable.

\section{Funding}

This study was supported by grants from the National Natural Science Foundation of China (nos. 81772384 and 81572174). The funders had no role in the study design, data collection and analysis, decision to publish, or preparation of the manuscript.

\section{Availability of data and materials}

The microarray data GSE8077 were downloaded from the GEO database in NCBI (http://www.ncbi.nlm.nih.gov/geo/).

\section{Authors' contributions}

LHZ and LHD participated in the design of this study and performed the statistical analysis. They both carried out the study and collected important background information. LHZ drafted the manuscript. All authors read and approved the final manuscript.

\section{Ethics approval and consent to participate} Not applicable.

\section{Consent for publication}

All authors read and approved the final manuscript and consented to publication.

\section{Competing interests}

The authors declare that they have no competing interest.

\section{Publisher's Note}

Springer Nature remains neutral with regard to jurisdictional claims in published maps and institutional affiliations.

Received: 19 April 2018 Accepted: 26 November 2018 Published online: 14 December 2018

\section{References}

1. Kiadaliri AA, Lohmander LS, Moradi-Lakeh M, Petersson IF, Englund M. High and rising burden of hip and knee osteoarthritis in the nordic region, 19902015. Acta Orthop. 2018;89:177-83.

2. Cross M, Smith E, Hoy D, Nolte S, Ackerman I, Fransen M, Bridgett L, Williams S, Guillemin F, Hill CL, Laslett LL, Jones G, Cicuttini F, Osborne R, Vos T, Buchbinder R, Woolf A, March L. The global burden of hip and knee osteoarthritis: estimates from the global burden of disease 2010 study. Ann Rheum Dis. 2014;73:1323-30.

3. Conaghan PG, Kloppenburg M, Schett G, Bijlsma JW. Osteoarthritis research priorities: a report from a eular ad hoc expert committee. Ann Rheum Dis. 2014;73:1442-5

4. Park J, Mendy A, Vieira ER. Various types of arthritis in the united states: prevalence and age-related trends from 1999 to 2014. Am J Public Health. 2018;108:256-8.

5. Glyn-Jones S, Palmer AJ, Agricola R, Price AJ, Vincent TL, Weinans H, Carr AJ. Osteoarthritis. Lancet. 2015;386:376-87.

6. da Costa BR, Reichenbach S, Keller N, Nartey L, Wandel S, Juni P, Trelle S. Effectiveness of non-steroidal anti-inflammatory drugs for the treatment of pain in knee and hip osteoarthritis: a network meta-analysis. Lancet. 2017;390:e21-33.

7. Jevsevar DS, Brown GA, Jones DL, Matzkin EG, Manner PA, Mooar P, Schousboe JT, Stovitz S, Sanders JO, Bozic KJ, Goldberg MJ, Martin WR 3rd, Cummins DS, Donnelly P, Woznica A, Gross L. The american academy of orthopaedic surgeons evidence-based guideline on: Treatment of osteoarthritis of the knee, 2nd edition. J Bone Joint Surg Am. 2013;95:1885-6.

8. Rausch Osthoff AK, Niedermann K, Braun J, Adams J, Brodin N, Dagfinrud H, Duruoz T, Esbensen BA, Gunther KP, Hurkmans E, Juhl CB, Kennedy N, Kiltz U, Knittle K, Nurmohamed M, Pais S, Severijns G, Swinnen TW, Pitsillidou IA, Warburton L, Yankov Z, Vliet Vlieland TPM. 2018 eular recommendations for physical activity in people with inflammatory arthritis and osteoarthritis. Ann Rheum Dis. 2018:77:1251-60.

9. Wang C, Schmid CH, Hibberd PL, Kalish R, Roubenoff R, Rones R, McAlindon $T$. Tai chi is effective in treating knee osteoarthritis: A randomized controlled trial. Arthritis Rheum. 2009;61:1545-53.

10. Ong KL, Anderson AF, Niazi F, Fierlinger AL, Kurtz SM, Altman RD. Hyaluronic acid injections in medicare knee osteoarthritis patients are associated with longer time to knee arthroplasty. J Arthroplast. 2016;31:1667-73.

11. Huynh C, Puyraimond-Zemmour D, Maillefert JF, Conaghan PG, Davis AM, Gunther KP, Hawker G, Hochberg MC, Kloppenburg M, Lim K, Lohmander LS, Mahomed NN, March L, Pavelka K, Punzi L, Roos EM, Sanchez-Riera L, Singh JA, Suarez-Almazor ME, Dougados M, Gossec L. Factors associated with the orthopaedic surgeon's decision to recommend total joint replacement in hip and knee osteoarthritis: An international cross-sectional study of 1905 patients. Osteoarthr Cartil. 2018;26:1311-8.

12. Kuttapitiya A, Assi L, Laing K, Hing C, Mitchell P, Whitley G, Harrison A, Howe FA, Ejindu V, Heron C, Sofat N. Microarray analysis of bone marrow lesions in osteoarthritis demonstrates upregulation of genes implicated in osteochondral turnover neurogenesis and inflammation. Ann Rheum Dis. 2017:76:1764-73.

13. Ramos YF, Bos SD, Lakenberg N, Bohringer S, den Hollander WJ, Kloppenburg M, Slagboom PE, Meulenbelt I. Genes expressed in blood link osteoarthritis with apoptotic pathways. Ann Rheum Dis. 2014;73:1844-53.

14. Zhang X, Yuan Z, Cui S. Identifying candidate genes involved in osteoarthritis through bioinformatics analysis. Clin Exp Rheumatol. 2016;34:282-90.

15. Liu J, Hao Y, Wang Y, Hu S, Xu K, Lu C. Candidate methylated genes in osteoarthritis explored by bioinformatics analysis. Knee. 2016;23:1035-43.

16. Parrish WR, Byers BA, Su D, Geesin J, Herzberg U, Wadsworth S, Bendele A, Story B. Intra-articular therapy with recombinant human gdf5 arrests disease progression and stimulates cartilage repair in the rat medial meniscus transection (mmt) model of osteoarthritis. Osteoarthr Cartil. 2017;25:554-60.

17. Gao H, Gui J, Wang L, Xu Y, Jiang Y, Xiong M, Cui Y. Aquaporin 1 contributes to chondrocyte apoptosis in a rat model of osteoarthritis. Int J Mol Med. 2016:38:1752-8.

18. Cheng J, Hu X, Dai L, Zhang X, Ren B, Shi W, Liu Z, Duan X, Zhang J, Fu X, Chen W, Ao Y. Inhibition of transforming growth factor beta-activated kinase 1 prevents inflammation-related cartilage degradation in osteoarthritis. Sci Rep. 2016:6:34497.

19. Appleton CT, Pitelka V, Henry J, Beier F. Global analyses of gene expression in early experimental osteoarthritis. Arthritis Rheum. 2007:56:1854-68.

20. Tripathi S, Pohl MO, Zhou Y, Rodriguez-Frandsen A, Wang G, Stein DA, Moulton HM, DeJesus P, Che J, Mulder LC, Yanguez E, Andenmatten D, Pache L, Manicassamy B, Albrecht RA, Gonzalez MG, Nguyen Q, Brass A, Elledge S, White M, Shapira S, Hacohen N, Karlas A, Meyer TF, Shales M, Gatorano A, Johnson JR, Jang G, Johnson T, Verschueren E, Sanders D, Krogan N, Shaw M, Konig R, Stertz S, Garcia-Sastre A, Chanda SK. Meta- and orthogonal integration of influenza "omics" data defines a role for ubr4 in virus budding. Cell Host Microbe. 2015;18:723-35.

21. Wadi L, Meyer M, Weiser J, Stein LD, Reimand J. Impact of outdated gene annotations on pathway enrichment analysis. Nat Methods. 2016;13:705-6.

22. Szklarczyk D, Franceschini A, Wyder S, Forslund K, Heller D, Huerta-Cepas J, Simonovic M, Roth A, Santos A, Tsafou KP, Kuhn M, Bork P, Jensen $\amalg$, von Mering C. String v10: Protein-protein interaction networks, integrated over the tree of life. Nucleic Acids Res. 2015;43:D447-52.

23. Cline MS, Smoot M, Cerami E, Kuchinsky A, Landys N, Workman C, Christmas R, Avila-Campilo I, Creech M, Gross B, Hanspers K, Isserlin R, Kelley R, Killcoyne S, Lotia S, Maere S, Morris J, Ono K, Pavlovic V, Pico AR, Vailaya A, Wang PL, Adler A, Conklin BR, Hood L, Kuiper M, Sander C, Schmulevich I, Schwikowski B, Warner GJ, Ideker T, Bader GD. Integration of biological networks and gene expression data using cytoscape. Nat Protoc. 2007; 2:2366-82.

24. Scardoni G, Petterlini M, Laudanna C. Analyzing biological network parameters with centiscape. Bioinformatics. 2009;25:2857-9.

25. Bader GD, Hogue CW. An automated method for finding molecular complexes in large protein interaction networks. BMC Bioinformatics. 2003;4:2.

26. Micrornas BDP. Genomics, biogenesis, mechanism and function. Cell. 2004; 116:281-97.

27. Wong $N$, Wang X. Mirdb: An online resource for microrna target prediction and functional annotations. Nucleic Acids Res. 2015;43:D146-52. 
28. Vlachos IS, Zagganas K, Paraskevopoulou MD, Georgakilas G, Karagkouni D Vergoulis T, Dalamagas T, Hatzigeorgiou AG. Diana-mirpath v3.0: Deciphering microrna function with experimental support. Nucleic Acids Res. 2015:43:W460-6.

29. Mapp PI, Avery PS, McWilliams DF, Bowyer J, Day C, Moores S, Webster R, Walsh DA. Angiogenesis in two animal models of osteoarthritis. Osteoarthr Cartil. 2008;16:61-9.

30. Liu J, Dai J, Wang Y, Lai S, Wang S. Significance of new blood vessels in the pathogenesis of temporomandibular joint osteoarthritis. Exp Ther Med. 2017; 13:2325-31

31. Walsh DA, McWilliams DF, Turley MJ, Dixon MR, Franses RE, Mapp PI, Wilson $D$. Angiogenesis and nerve growth factor at the osteochondral junction in rheumatoid arthritis and osteoarthritis. Rheumatology (Oxford). 2010; 49:1852-61

32. Murata M, Yudoh K, Masuko K. The potential role of vascular endothelial growth factor (vegf) in cartilage How the angiogenic factor could be involved in the pathogenesis of osteoarthritis? Osteoarthritis Cartilage. 2008;16:279-86.

33. Walsh DA, Bonnet CS, Turner EL, Wilson D, Situ M, McWilliams DF Angiogenesis in the synovium and at the osteochondral junction in osteoarthritis. Osteoarthr Cartil. 2007;15:743-51.

34. Hu W, Zhang W, Li F, Guo F, Chen A. Mir-139 is up-regulated in osteoarthritis and inhibits chondrocyte proliferation and migration possibly via suppressing eif4g2 and igf1r. Biochem Biophys Res Commun. 2016:474:296-302

35. O'Connell GD, Tan AR, Cui V, Bulinski JC, Cook JL, Attur M, Abramson SB, Ateshian GA, Hung CT. Human chondrocyte migration behaviour to guide the development of engineered cartilage. J Tissue Eng Regen Med. 2017;11:877-86.

36. Ntoumou E, Tzetis M, Braoudaki M, Lambrou G, Poulou M, Malizos K Stefanou N, Anastasopoulou L, Tsezou A. Serum microrna array analysis identifies mir-140-3p, mir-33b-3p and mir-671-3p as potential osteoarthritis biomarkers involved in metabolic processes. J Cell Biochem. 2017;9:127.

37. Luo H, Yao L, Zhang Y, Li R. Liquid chromatography-mass spectrometrybased quantitative proteomics analysis reveals chondroprotective effects of astragaloside iv in interleukin-1 beta-induced sw1353 chondrocyte-like cells. Biomed Pharmacother. 2017;91:796-802.

38. Volkert G, Jahn A, Dinkel C, Fahlbusch F, Zurn C, Hilgers KF, Rascher W, Hartner A, Marek I. Contribution of the alpha8 integrin chain to the expression of extracellular matrix components. Cell Commun Adhes. 2014;21:89-98.

39. Gong AX, Zhang JH, Li J, Wu J, Wang L, Miao DS. Comparison of gene expression profiles between dental pulp and periodontal ligament tissues in humans. Int J Mol Med. 2017;40:647-60.

40. Johnson K, Reynard LN, Loughlin J. Functional characterisation of the osteoarthritis susceptibility locus at chromosome 6 q14.1 marked by the polymorphism rs9350591. BMC Med Genet. 2015;16:81.

41. Jeyakumar V, Halbwirth F, Niculescu-Morzsa E, Bauer C, Zwickl H, Kern D, Nehrer S. Chondrogenic gene expression differences between chondrocytes from osteoarthritic and non-oa trauma joints in a $3 \mathrm{~d}$ collagen type hydrogel. Cartilage. 2017;8:191-8.

42. He P, Zhang Z, Liao W, Xu D, Fu M, Kang Y. Screening of gene signatures for rheumatoid arthritis and osteoarthritis based on bioinformatics analysis. Mol Med Rep. 2016;14:1587-93.

43. Kim JH, Jeon J, Shin M, Won Y, Lee M, Kwak JS, Lee G, Rhee J, Ryu JH, Chun $\mathrm{CH}$, Chun JS. Regulation of the catabolic cascade in osteoarthritis by the zinc-zip8-mtf1 axis. Cell. 2014;156:730-43.

44. Bonfante HL, Almeida CS, Abramo C, Grunewald STF, Levy RA, Teixeira HC. $\mathrm{Ccl} 2, \mathrm{cxcl} 8, \mathrm{cxc} 19$ and $\mathrm{cxcl} 10$ serum levels increase with age but are not altered by treatment with hydroxychloroquine in patients with osteoarthritis of the knees. Int J Rheum Dis. 2017;20:1958-64.

45. Snelling SJ, Bas S, Puskas GJ, Dakin SG, Suva D, Finckh A, Gabay C, Hoffmeyer P, Carr AJ, Lubbeke A. Presence of il-17 in synovial fluid identifies a potential inflammatory osteoarthritic phenotype. PLoS One. 2017;12:e0175109.

46. Miotla Zarebska J, Chanalaris A, Driscoll C, Burleigh A, Miller RE, Malfait AM, Stott B, Vincent TL. C $\mathrm{Cl}$ and $\mathrm{ccr} 2$ regulate pain-related behaviour and early gene expression in post-traumatic murine osteoarthritis but contribute little to chondropathy. Osteoarthr Cartil. 2017;25:406-12.
47. Raghu H, Lepus CM, Wang Q, Wong HH, Lingampalli N, Oliviero F, Punzi L, Giori NJ, Goodman SB, Chu CR, Sokolove JB, Robinson WH. Ccl2/ccr2, but not ccl5/ccr5, mediates monocyte recruitment, inflammation and cartilage destruction in osteoarthritis. Ann Rheum Dis. 2017;76:914-22

48. Unguryte A, Bernotiene E, Bagdonas E, Garberyte S, Porvaneckas N, Jorgensen C. Human articular chondrocytes with higher aldehyde dehydrogenase activity have stronger expression of col2a1 and sox9. Osteoarthr Cartil. 2016;24:873-82.

49. Hu G, Zhao X, Wang C, Geng Y, Zhao J, Xu J, Zuo B, Zhao C, Wang C, Zhang $X$. Microrna-145 attenuates tnf-alpha-driven cartilage matrix degradation in osteoarthritis via direct suppression of mkk4. Cell Death Dis. 2017;8:e3140

50. Si HB, Zeng Y, Liu SY, Zhou ZK, Chen YN, Cheng JQ, Lu YR, Shen B. Intra-articular injection of microrna-140 (mirna-140) alleviates osteoarthritis (oa) progression by modulating extracellular matrix (ecm) homeostasis in rats. Osteoarthr Cartil. 2017;25:1698-707.

51. Kong R, Gao J, Si Y, Zhao D. Combination of circulating mir-19b-3p, mir-122$5 p$ and mir-486-5p expressions correlates with risk and disease severity of knee osteoarthritis. Am J Transl Res. 2017;9:2852-64.

52. Sun HY, Hu KZ, Yin ZS. Inhibition of the p38-mapk signaling pathway suppresses the apoptosis and expression of proinflammatory cytokines in human osteoarthritis chondrocytes. Cytokine. 2017;90:135-43.

53. Akhtar N, Haqqi TM. Microrna-199a* regulates the expression of cyclooxygenase-2 in human chondrocytes. Ann Rheum Dis. 2012;71:1073-80.
Ready to submit your research? Choose BMC and benefit from:

- fast, convenient online submission

- thorough peer review by experienced researchers in your field

- rapid publication on acceptance

- support for research data, including large and complex data types

- gold Open Access which fosters wider collaboration and increased citations

- maximum visibility for your research: over $100 \mathrm{M}$ website views per year

At BMC, research is always in progress.

Learn more biomedcentral.com/submissions 